\title{
Protocollo della seduta straordinaria della Camera medica svizzera
}

Giovedì, 28 ottobre 2004, dalle ore 9.30 alle 17.00, centro dei congressi di Berna

Annamaria Müller Imboden, segretaria generale della FMH

Inizio: ore 9.40.

\section{Benvenuto, comunicazioni}

Il presidente della FMH, Jacques de Haller, saluta i partecipanti e costata che il quorum è raggiunto e che quindi l'assemblea è regolarmente costituita. Presenta la nuova composizione del Comitato centrale e ricorda che un'azione costruttiva è possibile solamente con una collaborazione con i delegati del corpo medico e dei suoi organi. Per lui sono estremamente importanti trasparenza e apertura, ma anche dei rapporti con gli altri che si basino sul rispetto reciproco. De Haller fa delle osservazioni d'ordine tecnico e organizzativo e saluta gli ospiti della Camera medica di oggi: si tratta di Urs Kayser, come anche André Bürki e Stephan Thommen del gruppo di consulenti in gestione di associazione B'VM. Sono scusati i membri del consiglio Ludwig Heuss e Yves Guisan, che raggiungeranno la riunione solamente nel pomeriggio. De Haller saluta, inoltre, la nuova responsabile della segreteria del Comitato centrale, Gabriela Kaufmann, che succede a Jessica Soldati che si è lanciata in un nuovo challenge professionale.

Sono designati come scrutatori: Suzanne von Blumenthal, Marcel Butz, Hermann Engel, Felix Eymann, Guy Jenny, Beat de Roche, Pierre-Alain Schneider, Edouard Stauffer, Christian Stettler, Hans-Anton Vogel.

\section{Proposta N. 1/1 (Comitato centrale)}

richiede dei complementi all'ordine del giorno, e più precisamente: la revisione degli Statuti in vista del diritto dell'appena fondata FMCH a sedere negli organi della FMH (punto N. 3 dell'ordine del giorno), l'elezione per la sostituzione di un membro della Commissione d'assessment LAINF/AM/AI (punto N. 4 dell'ordine del giorno) come anche la consultazione su un numero di pronto soccorso a tre cifre valido in tutta la Svizzera. La proposta viene approvata all'unanimità, senza che vengano contati i voti, senza voti contrari e senza astensioni.
Jacques de Haller fa inoltre notare che il punto dell'ordine del giorno «Dati, demografia e qualità» è stato soppresso, in quanto al momento non si dispone ancora di informazioni concrete.

\section{Comitato centrale: informazioni generali}

Nel Bollettino dei medici svizzeri [1] è stata pubblicata la distribuzione dei campi di attività. Ogni campo di attività è ora composto da un responsabile e da diversi collaboratori del Comitato centrale. In questo modo si intende promuovere lo spirito di gruppo e creare delle competenze. Il Comitato centrale, inoltre, presenta le sue riflessioni sulla futura strategia da adottare. Queste mirano a rinforzare la posizione della FMH nel settore della politica sanitaria in modo da renderla più presente e più attiva nelle discussioni d'ordine politico, scientifico ed etico. La FMH deve adottare una posizione chiara e seguire una line precisa.

\section{La revisione degli Statuti}

Il Comitato centrale ha preso nota con grande soddisfazione della soluzione trovata per le società di chirurgia. In questo senso si sono espressi insieme in un editoriale de Haller/Laffer [2].

3a) Ammissione della FMCH (Foederatio Medicorum Chirurgicorum Helvetica) negli organi della FMH come organizzazione con diritto di essere consultata

Proposta N. 3a/1 (Urban Laffer, FMCH, Comitato centrale)

fa notare che la FMCH è l'organizzazione mantello di tutte le società mediche di chirurgia e della Società svizzera di anestesiologia e rianimazione SSAR, e deve essere integrata come organizzazione succeditrice della FMS (Foederatio Medicorum Scrutantium) negli organi della FMH. 
Conformemente all'art. 25, cpv. 2 degli Statuti della FMH, la FMCH ottiene in questo modo il diritto di consultazione e di proposta, ma non il diritto di voto e di eleggibilità. Come le altre organizzazioni corrispondenti, viene iscritta nell'elenco nell'appendice IIII degli Statuti della FMH. La proposta viene messa in votazione insieme alla proposta $3 b / 1$ e senza che vengano conati i voti viene approvata all'unanimità senza voti contrari o astensioni.

\section{3b) Diritto della FMCH di sedere nella CPA}

Proposta N. 3b/1 (Urban Laffer, FMCH)

richiede il diritto per la FMCH di sedere nella Commissione per il perfezionamento professionale e l'aggiornamento (CPA) e viene approvata conformemente alla votazione raggruppata con la proposta $3 a / 1$.

\section{Commissione d'assessment LAIN/AM/AI}

La Commissione d'assessment ha concluso i suoi lavori il 31.10.2004. Per motivi statutari si deve tuttavia eleggere per i giorni rimanenti un successore a Anne-Claude Froidevaux che ha presentato le dimissioni. Candidato è Philippe Rheiner. Il Comitato centrale presenta la mozione d'ordine, secondo cui l'elezione deve avvenire a mano levata. La proposta viene approvata senza che vengano contati i voti, con un voto contrario e senza astensioni. Si passa quindi all'elezione e Philippe Rheiner viene eletto nella Commissione d'assessment senza che vengano contati i voti, con un voto contrario e un'astensione. Si ringrazia qui caldamente Anne-Claude Froidevaux per il suo impegno.

\section{Tariffe, valore intrinseco}

Il responsabile del settore Tariffe, Franco Muggli, fa un breve riepilogo dei diversi compiti impegnativi e dà la possibilità ad ogni esponente di fare un rapporto sui compiti e sui suoi progetti.

Urs Stoffel informa sui lavori dell'ufficio della neutralità dei costi. L'ufficio ha iniziato la sua attività ai primi di quest'anno e nel frattempo ha già effettuato due serie di correzioni del valore del punto. In proposito si sottolinea nuovamente come fosse e sia tuttora decisivo disporre di proprie cifre. L'affermazione «ciò nonostante il valore del punto è stato diminuito» è errata, si trattava piuttosto del fatto che con le cifre proprie è stato possibile evitare delle riduzioni maggiori. Poiché si tratta della regolamentazione della neutralità dei costi, i ritardi nell'applicazione rappresentano un problema reale. I partner tariffari hanno quindi scelto come soluzione un modello di correzione nel quale vengono integrati diversi parametri di flusso (le cosiddette "curve d'effetto» e le «curve d'eliminazione»). Il canton Ginevra assume nella procedura di correzione una posizione speciale, da una parte perché esiste una comunità contrattuale tra i medici con studio proprio e gli ospedali, e dall'altra il Governo cantonale ginevrino non riconosce nella sua procedura d'autorizzazione i risultati dell'ufficio della neutralità dei costi. Gli ospedali sono in genere in grande ritardo per quel che concerne la neutralità dei costi. Di conseguenza l'UFSP ha ora richiesto a $\mathrm{H}+\mathrm{di}$ proporre un modello di soluzione. Per la FMH è decisivo che questo modello non costituisca in nessun modo un pregiudizio per i medici che praticano in studio medico.

Olivier Kappeler ha informato sulla banca dati del valore intrinseco. Al momento della ripresa del progetto da parte del Comitato centrale nella sua nuova composizione, questi ha dovuto affrontare un ultimatum della relativa commissione paritetica per il controllo della banca dati sul valore intrinseco e sulle unità tariffarie, che richiedeva la realizzazione entro fine settembre di una fase di test, altrimenti i partner tariffari minacciavano di occuparsi essi stessi della gestione del valore intrinseco. Investendo molto tempo e lavoro si è riusciti ad osservare i termini fissati. Gli assicuratori non si sono però più mostrati interessati alla fase test. Hanno prolungato l'ultimatum sino a fine anno ed entro questo termine attendono che i dati vengano convalidati. La FMH si è fissata come obiettivo di permettere ai medici di gestire autonomamente i loro dati sul valore intrinseco mediante un accesso sicuro, in modo che sia possibile evitare nuovi rilevamenti che richiedono molto tempo. Al momento sono necessari ancora degli accertamenti sul significato giuridico degli attestati numerici di valore intrinseco e della possibilità di una convalida diretta («up front») delle fatture tramite i centri fiduciari.

Marie-Christine Peter informa sui lavori della commissione tariffaria CPI (commissione paritetica di interpretazione) e della CPT (commissione paritetica tariffaria), che sono state incaricate dal Comitato di direzione TARMED. Mentre la CPI si occupa principalmente di questioni relative all'applicazione delle tariffe, la CPT si occupa dell'adattamento della struttura tariffaria e delle regole d'applicazione. In generale è stato constato che la comunicazione e il trasferimento di informazioni tra gli organi ovvero i rappresentanti della FMH e delle società mediche speciali- 
stiche non sono ancora ottimali. Il gruppo tariffe del Comitato centrale (Muggli, Kappeler, Peter) tiene conto delle diverse domande e cercherà delle soluzioni insieme al servizio tariffario. Osservazione di carattere generale: il servizio tariffario della FMH rimane il principale interlocutore per tutte le questioni relative alle tariffe. Provocano delle discussioni anche singoli punti tariffari, in particolare le posizioni per il pronto soccorso.

François Bossard parla della fase di stabilizzazione dei costi per caso per il settore LAINF/AM/ AI che giunge alla fine. La fase non viene prolungata, ma si è dovuta accettare un diminuzione del valore del punto tassa di 8 centesimi. Attualmente sono in corso ai livelli massimi delle discussioni tra FMH e CTM sul seguito delle operazioni. Diversi rappresentanti del corpo medico chiedono che si agisca in modo duro e inflessibile nei confronti degli assicuratori e li accusano di comportarsi in modo sleale per aver dato degli ultimatum e utilizzato delle cifre non accettabili. Si minaccia anche il boicottaggio se negli ospedali e negli studi medici si dovessero applicare dei valori tariffari LAINF diversi.

Alla fine delle presentazioni e dell'animata discussione Franco Muggli ringrazia tutti coloro che hanno partecipato ai negoziati, che hanno dato il loro sostegno e che ne hanno subito le conseguenze. Costata che è stato un compito quanto mai difficile quello che lui, i suoi colleghi e le sue colleghe del Comitato centrale hanno dovuto affrontare da metà anno. Sottolinea comunque nuovamente che il Comitato centrale e lui stesso sono pronti ad assumersi la responsabilità e a rispondere di eventuali conseguenze. La Camera dei medici manifesta il suo ringraziamento con un applauso.

Proposta N. 5/1 (Guy Jenny, Società svizzera di oftalmologia)

richiede che vengano prolungati i meccanismi di sorveglianza nella TARMED (stabilizzazione dei costi per caso e neutralità dei costi) solamente con l'accordo della Camera dei medici e che si esiga la trasparenza dei costi da parte degli assicuratori in modo che i loro costi non subiscano, proporzionalmente, un aumento superiore a quello dei fornitori di prestazioni. L'autore della proposta è cosciente del fatto che le esigenze poste agli assicuratori non possano essere garantite con documenti. Si tratta tuttavia di prendere una posizione chiara e di farlo sentire alla controparte. In questo senso la proposta viene approvata senza che vengano contati i voti con un voto contrario e diverse astensioni. La proposta viene trasmessa al G7 che conduce le trattative.
Proposta N. 5/2 (Marcus Lütolf, SGR-SSR), che domanda che vengano iniziate immediatamente le trattative in vista di prolungare le misure d'emergenza per i radiologi che hanno uno studio, viene ritirata, perché per il momento la questione è regolata (sino al 30 giugno 2005).

\section{Proposta N. 5/3 (Marcus Lütolf, SGR-SSR)}

richiede dalla FMH un'analisi a livello nazionale dei costi conformemente alla TARMED per il periodo dal $1^{\circ}$ gennaio 2002 al 31 dicembre 2004 su tutti i settori della neutralità dei costi (ambulatoriale, ambulatoriale ospedaliero e ospedaliero) secondo dei criteri differenti, in diverse varianti di valutazione e chiede anche che i risultati siano pubblicati a metà febbraio 2005 nel Bollettino dei medici svizzeri. L'analisi dovrà poi essere ripetuta semestralmente. Per quanto la direzione di questa proposta venga accolta positivamente da tutti, la discussione che segue mette in luce le difficoltà considerevoli che comporterebbe un'applicazione alla lettera:

- i termini per disporre di dati consolidati e garantiti sono troppo brevi;

- la valutazione può essere fatta solamente per i medici con studio, perché i dati relativi agli ospedali vengono gestiti da $\mathrm{H}+$;

- per la trasmissione di dati alla FMH è necessaria un'autorizzazione da parte del proprietario dei dati;

- la classificazione richiesta violerebbe il principio della protezione dei dati.

Per i motivi sopraindicati la proposta viene quindi modificata nel senso che il Comitato centrale possa verificare l'applicabilità delle valutazioni e elabori una proposta, che tenga conto del senso e dello spirito della presente proposta. In questa proposta deve esserci anche un'informazione adeguata, in quanto i media assumono un ruolo non indifferente. La data di pubblicazione viene spostata alla fine della fase di neutralità dei costi. In questa forma modificata la proposta viene approvata senza che vengano contati i voti, con due voti contrari e qualche astensione.

\section{Proposta N. 5/4 (Marcus Lütolf, SGR-SSR)}

concerne la composizione, l'incarico e le procedure di lavoro dell' «Ufficio TARMED FMH» e chiede un coinvolgimento adeguato delle società mediche specialistiche in seno a quest'ultimo. In considerazione del fatto che la riorganizzazione degli organi tariffari è ancora in corso, la proposta viene trasmessa senza votazione al Comitato centrale perché la tratti. 


\section{Riforma delle strutture della FMH: quo vadis?}

I rappresentanti del gruppo di consulenti in gestione d'associazione B'VM, André Bürki e Stephan Thommen, presentano il progetto e le diverse fasi previste. Il calendario, così come è stato previsto, è molto ambizioso, ma in considerazione della situazione anche giustificato. Suo obiettivo è di elaborare entro la fine del 2005 delle proposte concrete. Di importanza primaria è il fatto che tutte le persone coinvolte, compresi i membri della FMH, non si accontentino solo di auspicare il cambiamento, ma anche lo accettino. Uno dei fattori critici di successo è qui una visione comune sull'essenza e sugli obiettivi dell'associazione professionale. Il Comitato centrale tratta quindi questa problematica come una delle sue attività principali. È inoltre previsto di far partecipare alla riflessione anche i membri della FMH tramite dei questionari. I delegati auspicano che a tutti i livelli l'informazione sia rapida e dettagliata. Le altre domande concernono gli organi del progetto e le conseguenze finanziarie. Poiché a parte il Comitato di direzione non esiste attualmente nessun altro organo di progetto e le finanze, fatta eccezione per l'ambito dei costi, sono pure oggetto di analisi esatte, per una risposta dettagliata sarà necessario attendere ancora. In effetti non erano state previste sulle uscite specifiche relative alla riforma delle strutture né del budget 2004 né in quello per il 2005.

\section{Numero di pronto soccorso a tre cifre valido in tutta la Svizzera}

\section{Proposta N. 7/1 (Christoph Ramstein, VEDAG)}

chiede al Comitato centrale di informarsi - a proposito dell'introduzione in tutta la Svizzera di un numero di pronto soccorso a tre cifre - sui tempi necessari per la sua realizzazione e sulle relative conseguenze finanziarie che questo rappresenta. La proposta è stata provocata dalla situazione assai insoddisfacente e estremamente eterogenea che regna nei diversi cantoni e nelle varie regioni. Parte anche dal principio che la garanzia di un servizio di pronto soccorso ben funzionante da parte del corpo medico svizzero sia un elemento importante per le relazioni pubbliche. Marc Müller, presidente della piattaforma soccorso della FMH, spiega che la piattaforma si sta occupando già da un certo tempo proprio di questo argomento, poiché la Società medica ginevrina si è rivolta al Comitato centrale con domande analoghe già un anno fa. La proposta viene formulata in modo nuovo, e cioè come una proposta della Camera dei medici alla piattaforma Soccorso e trasmessa a questo organo senza che vengano contati i voti, con due voti contrari e due astensioni.

\section{Votazione popolare federale del 28 novembre 2004: votazione sulla Legge federale relativa alla ricerca sulle cellule staminali embrionali}

Ursula Steiner, vicepresidentessa della FMH e responsabile del settore Public Health, riassume il progetto e spiega che cosa, secondo a legge, sarà permesso e che cosa vietato. La legge si basa sul cosiddetto «Modello di rispetto», secondo il quale un'utilizzazione limitata e specifica di embrioni in soprannumero «destinati alla morte» sia eticamente sostenibile. Urs Kayser presenta le posizioni degli avversari, che principalmente si basano sul carattere dubbio della ricerca sulle cellule staminali di per sé e sulla protezione della vita dal momento della concezione.

Proposta N. 8/1 (Jürg Schlup, Società medica del Canton Berna)

chiede un sì sulla legge sulla ricerca sulle cellule staminali, poiché i controlli sono più importanti dei divieti e non si può mettere in pericolo la ricerca in Svizzera. Dopo una discussione intensa e differenziata la Camera medica approva la proposta con 131 voti favorevoli, 8 voti contrari e 9 astensioni. Tuttavia si chiede che nella redazione dei comunicati stampa si tenga conto in modo appropriato dei pareri differenti.

\section{Medical Education: situazione e progetti in corso}

Max Giger, responsabile della settore Formazione medica, informa sulla situazione e sugli sviluppi futuri in questo settore. Secondo quanto auspicato dai responsabili politici, lo studio della medicina deve essere integrato nel rimodellamento del paesaggio universitario. A questo scopo è stato creato un organo composto da rappresentanti delle cerchie coinvolte, quindi anche della FMH. Obiettivo è rendere più trasparente e più efficiente lo studio della medicina riducendo nel contempo i costi. Competente è qui il «Gruppo Kleiber», di cui Max Giger presenta brevemente la composizione e i compiti. Giger riassume così:

- L'immagine della professione del medico deve adattarsi alle necessità della società. 
- Non può essere evitata una discussione su una regolamentazione del numero di medici.

- Nel perfezionamento professionale viene richiesto un incremento continuo della qualità.

- Si impegna la creazione di centri medici di competenze.

Nel corso della discussione che segue si fa tra l'altro notare che l'idoneità allo svolgimento della professione non concerne solamente l'università, ma anche e soprattutto la pratica quotidiana.

\section{Revisione della LAMal: situazione tra le sessioni parlamentari, prospettive}

Yves Guisan, vicepresidente della FMH e responsabile del settore LAMal, informa sull'avanzamento delle consultazioni parlamentari. Il prolungamento del blocco delle autorizzazioni ha sollevato un certo polverone. Yves Guisan constata che alle Camere il sostegno per i problemi del corpo medico è piuttosto scarso. Ora la FMH si è quindi attivata e ha presentato alla Commissione del Consiglio degli Stati (CSS) una proposta alternativa per eliminare l'obbligo di contrarre. Questa proposta è stata trasmessa all'amministrazione affinché la studi più dettagliatamente. Yves Guisan pensa che sia un segnale positivo.

\section{Proposta N. 10/1 (Jürg Schlup,}

\section{Società medica del Canton Berna)}

chiede che venga rinnovata la decisione di lanciare un referendum contro la limitazione della libera scelta del medico ovvero l'eliminazione dell'obbligo di contrarre. Se in Parlamento il progetto dovesse subire delle modifiche importanti per il corpo medico, il Comitato centrale dovrebbe essere autorizzato a convocare d'urgenza una Camera medica, senza che debba osservare i termini di convocazione previsti dagli Statuti conformemente all'art. 31. La proposta viene completata con la condizione che nel caso si ricorresse al referendum la FMH deve fondare un comitato di referendum oppure aderire ad un comitato del genere, in modo che si possa ottenere il sostegno di altri gruppi. Con questa modifica la proposta viene approvata con 129 voti favorevoli, 3 contrari e nessuna astensione.

Proposta N. 10/2 (Peter Studer, Oscar Matzinger, ASMAC)

richiede fondamentalmente la stessa cosa della proposta N. 10/1. Contiene in più un passaggio sul comitato di referendum, che viene integrato nella proposta qui sopra. La proposta viene quindi riunita alla proposta N. 10/1.

L'ASMAC sottolinea di opporsi del tutto all'abolizione dell'obbligo di contrarre. Con il modello positivo da lui proposto vorrebbe però liberarsi dal ruolo del «Neinsager». La diffusione di questo modello ha creato frattanto all'interno del corpo medico un certo disagio ed è stato percepito come un'azione «da sola» dell'ASMAC. Yves Guisan desidererebbe sapere che cosa pensi la Camera della proposta di lasciare l'obbligo di contrarre solo per le reti dei medici e di allentarlo in altri settori. Da una votazione consultiva tra $i$ delegati risulta che quest'idea viene respinta dalla maggioranza, con 4 voti favorevoli e 9 astensioni. Jacques de Haller schizza brevemente le proposte alternative della FMH, per le quali in primo piano figura l'attenzione sulle prestazioni fornite dai medici. L'art. 36a LAMal deve essere riformulato in tal senso, in modo che l'autorizzazione dell'attività a carico dell'assicurazione malattia sociale possa essere resa dipendente da criteri relativi alla qualità e alla redditività. I partner tariffari sono stati incaricati di elaborare dei criteri unitari per tutto il paese, criteri che dovrebbero essere approvati dal Consiglio federale. Se non si riuscisse a trovare un accordo il Consiglio federale dovrebbe fissare i criteri. E questo sulla base di proposte elaborate da una commissione composta dai rappresentanti del corpo medico, degli assicuratori, dei cantoni e degli assicurati. I delegati della Camera medica si mostrano favorevoli a queste proposte anche se per quel che concerne l'elaborazione dei criteri non mancano le domande e numerosi dubbi.

\section{Swiss DRG}

Pierre-François Cuénoud presenta il progetto SwissDRG. Si tratta dell'elaborazione di un sistema di rimunerazione basato sulle prestazioni per le degenze in ospedale per cure sanitarie somatiche acute. Il sistema si basa sul DRG americano, «Diagnosis Related Groups». Si tratta di un progetto tra partner nel quale oltre alla FMH sono coinvolti anche $\mathrm{CDS}, \mathrm{H}+$, santésuisse e la CTM come membri a parte intera. Per la realizzazione del progetto sono previsti 3 anni e un budget leggermente superiore a 3 milioni di franchi. Cuénoud costata che gli interessi sono molto controversi e che si può dubitare molto che tutti i partner siano veramente interessati alla realizzazione del progetto. Considerando ciò ci si pone giustamente la domanda se la FMH debba essere membro a parte intera o se - analogamente 
all'Associazione svizzera infermiere e infermieri (ASI) - non dovrebbe mirare ad un ruolo di membro passivo oppure ad assumere un ruolo di osservatore. I rappresentanti della FMH propongono di rimanere nel progetto, almeno sino a quando l'incarico per il progetto pattuito e il budget vengano osservati. Vengono sollevate delle questioni che concernono l'attività del medico e non sarebbe politicamente giudizioso abbandonare il progetto al momento attuale. Se il progetto minacciasse di degenerare in una seconda «TARMED», la FMH deve poter riservarsi il diritto di ritirarsi. Di importanza essenziale è il fatto che i gruppi direttamente coinvolti all'interno del corpo medico, soprattutto l'Associazione medici dirigenti ospedalieri svizzeri, la FMCH e la SSMI partecipino attivamente a questo progetto. A questo scopo ci deve essere uno scambio regolare di informazioni; si sta preparando una relativa proposta ad hoc. Le prestazioni psichiatriche non vengono per ora toccate, dal sistema di rimunerazione (DRG), poiché questo include solamente le cure per le malattie somatiche, gli infortuni e il loro trattamento.

\section{Varie}

De Haller ringrazia molto cordialmente Jessica Soldati, la segretaria uscente del Comitato centrale per il suo impegno e il lavoro svolto in un periodo quanto mai tumultuoso e le porge i migliori auguri di successo per la sua carriera professionale.

Fine della seduta: ore 17.00. 